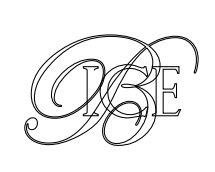

\title{
EL FONDO MONETARIO INTERNACIONAL EN LA ENCRUCIJADA La Decimoquinta Revisión General de Cuotas y otras propuestas de reforma en un sistema financiero internacional en transición
}

En este artículo se analiza la situación en el momento presente del Fondo Monetario Internacional como institución en términos de recursos y gobernanza, junto con unas referencias puntuales a los principales mensajes sobre la situación de la economía global de las últimas asambleas de octubre de 2018. Para ello, el foco se centra en dos propuestas recientes de reforma del Fondo desde el ámbito académico (última edición del Geneva Report de 2018) y desde el G20 (informe presentado por el Eminent Persons Group en octubre de 2018) y, particularmente, en los principales aspectos de la Decimoquinta Revisión General de Cuotas de la institución, actualmente en curso, y que ha de ser concluida como muy tarde en las asambleas anuales de octubre de 2018. Del resultado de este proceso de reforma depende en gran medida que el FMI permanezca en el centro de la red de seguridad del sistema financiero internacional.

Palabras clave: FMI, fórmula, derechos especiales de giro, NAB, préstamos bilaterales, reasignación de cuotas.

Clasificación JEL: F00, F02.

\section{Introducción}

Las reuniones anuales conjuntas de las Juntas de Gobernadores del Fondo Monetario Internacional (FMI) y del Grupo del Banco Mundial (GBM), que reúnen a los ministros de Finanzas y Economía y a los gobernadores

\footnotetext{
* Secretaría General del Tesoro y Financiación Internacional, Ministerio de Economía y Empresa. Este artículo ha sido elaborado por Ana Fornells de Frutos y por María Mateo Feito.

Versión de noviembre de 2018.
}

de los bancos centrales de los 189 países miembros de ambas instituciones, tienen lugar todos los meses de septiembre u octubre'. En paralelo a las reuniones oficiales, también se celebran eventos a los que acuden representantes del sector privado, miembros del mundo académico y otros representantes de la sociedad civil con el objetivo de debatir temas de actualidad económica.

\footnotetext{
1 La asamblea anual de este año tuvo lugar en Bali Nusa Dua, Indonesia, del 8 al 14 de octubre de 2018.
} 
Durante estos encuentros anuales se reúne el Comité Monetario y Financiero Internacional ${ }^{2}$, que negocia un comunicado en el que se dan orientaciones estratégicas sobre las políticas y operaciones del Fondo Monetario Internacional y se hace una valoración sobre las perspectivas para la economía internacional y las políticas económicas y reformas que los países deberían adoptar para hacer frente a los desafíos o riesgos identificados.

A la hora de hacer la lectura de los comunicados del Comité Monetario y Financiero Internacional hay que tener en cuenta que son negociados y acordados entre los 24 miembros que representan a los 189 países del FMI, por lo que se trata de un lenguaje de consenso que tiene que acomodar la postura, a veces divergente, de todos los países, pero que es muy representativo, ya que refleja la visión común de los Estados en temas de economía internacional.

\section{Perspectivas sobre la economía mundial}

La elaboración de informes sobre la coyuntura mundial es una de las principales labores del FMI, y son tres los principales informes que publica: las perspectivas económicas mundiales (World Economic Outlook, WEO), el informe global de estabilidad financiera (Global Financial Stability Report, GFSR) y el Monitor Fiscal (Fiscal Monitor).

En su informe de perspectivas económicas mundiales de octubre de 2018 (WEO), se estima que el crecimiento económico mundial será del 3,7 por 100 , tanto en 2018 como en 2019 , lo

2 El Comité Monetario y Financiero Internacional tiene un tamaño y una composición idénticos al Directorio Ejecutivo del FMI, con 24 miembros que representan al conjunto de los 189 países del FMI. que supone una revisión a la baja de 0,2 puntos porcentuales respecto a las proyecciones de abril.

Estas cifras representan aún un crecimiento sólido pero cada vez más desigual, en un contexto en el que se han materializado algunos de los riesgos apuntados en el WEO de abril, como el aumento de las tensiones comerciales y la huida de capitales de las economías emergentes con fundamentos económicos menos sólidos y mayores incertidumbres políticas. Además, se apunta a la normalización de la política monetaria por los países desarrollados como uno de los principales riesgos en caso de que se produzca de forma abrupta. En concreto, el crecimiento en EEUU continúa a buen ritmo, mientras que ha sido revisado a la baja para la zona euro, Japón, el Reino Unido, Argentina, Brasil, India, Irán y Turquía.

En esta misma línea, en los párrafos iniciales del comunicado del Comité Monetario y Financiero Internacional relativos a la interpretación sobre la situación de la economía global, se produce un importante cambio en el tono respecto al comunicado de abril. Así, se mantiene la afirmación de que el crecimiento económico mundial continúa siendo sólido, pero mientras que en el comunicado de abril se afirmaba que el crecimiento económico era cada más generalizado, en octubre se hace un llamamiento a la cautela porque dicho crecimiento pasa a ser cada vez más desigual, y en un contexto económico que ha empeorado. En concreto, hay mayores incertidumbres y algunos de los riesgos previamente identificados ya se han materializado, como el aumento de las tensiones comerciales y geopolíticas o el endurecimiento de las condiciones financieras que afectan en particular a muchas de las economías emergentes y en desarrollo. Respecto a las recomendaciones, el comunicado defiende que durante esta $\square$ 
fase expansiva del ciclo económico se impulsen políticas y reformas que favorezcan el crecimiento a medio plazo y fortalezcan los fundamentos económicos de los países para que estén mejor preparados para afrontar futuras crisis. Por otro lado, también se hace una mención específica a la necesidad de intensificar la cooperación internacional para hacer frente a los desafíos comunes. Estas recomendaciones son similares a las incluidas en los últimos comunicados semestrales del Comité Monetario y Financiero Internacional.

\section{Propuestas para reformar el Fondo Monetario Internacional}

Ante el contexto de fuerte incertidumbre en la que nos encontramos, se hace especialmente relevante contar con un FMI sólido, capaz de ejercer la labor para la que fue creado: garantizar la estabilidad financiera. $Y$ son numerosos los expertos que reclaman la necesidad de acometer reformas que faciliten que la institución pueda ejercer este papel. Sin perjuicio de otras medidas que puedan tomarse a medio plazo, la Decimoquinta Revisión General de Cuotas del FMI es una importante herramienta para avanzar en este sentido.

La propia directora gerente del Fondo Monetario Internacional, Christine Lagarde, durante las pasadas asambleas anuales de octubre de 2018, hizo un llamamiento para que los países doten al FMI de suficientes recursos para que pueda llevar a cabo adecuadamente sus funciones de préstamo, supervisión y fortalecimiento de las capacidades. En concreto, lo justifica por el nuevo contexto económico, en el que los países están cada vez más interconectados, así como en la necesidad de que, para hacer frente a los riesgos, las políticas internas se complementen con una red de seguridad financiera mundial en la que el FMI debe seguir jugando un papel central.

Sobre la gobernanza del FMI, en este artículo comenzaremos abordando las propuestas planteadas en dos informes publicados en 2018 , la última edición del denominado Informe de Ginebra (Geneva Report) y el informe del grupo de personas de reconocido prestigio (Eminent Persons Group) del G20, y posteriormente analizaremos la Decimoquinta Revisión General de Cuotas del FMI.

\subsection{El Informe de Ginebra}

Con carácter anual, el centro de investigación de política económica (The Centre for Economic Policy Research) y el Centro Internacional de Estudios Monetarios y Bancarios (The International Center for Monetary and Banking Studies) publican un informe sobre la economía mundial, el conocido como Informe de Ginebra. El primer informe fue publicado en 1999 y ahora en 2018 el mismo grupo de autores se ha vuelto a juntar para analizar los cambios experimentados por la economía global y el FMI bajo el título «La reforma del FMI, agenda inacabada».

Aunque algunos de los debates continúan siendo los mismos que hace diez años, como la discusión sobre la necesidad de dotar al FMI con recursos suficientes, se ha producido un importante cambio en el orden económico mundial, especialmente por el incremento del peso de China y la consiguiente incertidumbre sobre cómo va a ejercer este país su creciente poder en los diferentes foros e instituciones internacionales. En caso de que China se acabe convirtiendo en el país con la mayor cuota en el Fondo, habría que trasladar la sede a $\triangleright$ 
este Estado, como establecen los estatutos del FMI, lo que se traduciría en una mayor influencia de este país en el funcionamiento y toma de decisiones del FMI. Por el contrario, si no se llega a dar este caso, habría que hacer un ajuste adicional al sistema de cuotas para aproximarlo en mayor medida al peso de cada uno de los países en la economía internacional. Es probable que China acabe creando una red de seguridad financiera internacional paralela, como ha impulsado en gran medida en el ámbito de los bancos multilaterales de desarrollo con la creación del Banco Asiático de Inversión en Infraestructura o en el propio ámbito del sistema financiero internacional a través de los acuerdos de swap bilaterales cerrados por el banco central chino con un número creciente de bancos centrales de otros países.

La proliferación de los acuerdos financieros regionales ha sido otro de los cambios experimentados en los últimos años. El FMI deberá negociar acuerdos formales con estos convenios financieros regionales para concretar el sistema de cooperación entre ambos. En concreto, estos acuerdos deberán incluir un reparto claro de responsabilidades y un sistema de resolución de diferencias.

\subsubsection{Propuestas de reforma}

El informe de Ginebra propone la reforma del sistema de gobernanza del FMI y de sus instrumentos de préstamo. Los autores consideran que ambas reformas tienen que ir de la mano, ya que para poder gestionar los nuevos instrumentos es imprescindible que las decisiones operativas las adopte un comité de gestión que debe actuar como un banco central moderno, es decir, tiene que ser independiente y actuar siguiendo un mandato concreto que debe acordar la Junta de Gobernadores y en el que se establecerán los objetivos concretos que el comité de gestión ha de alcanzar, según un orden de priorización determinado.

\subsubsection{Nuevo sistema de gobernanza}

Se propone sustituir el Directorio Ejecutivo residente que existe en la actualidad, y que es el encargado de adoptar las decisiones operativas, por un directorio no residente. Con este nuevo sistema de gobernanza, las decisiones operativas y de políticas se delegarían en un comité de gestión.

Para justificar esta propuesta, el informe utiliza dos argumentos. Por un lado, la mayor legitimidad que se conseguiría, ya que en un directorio no residente podrían participar altos funcionarios de los ministerios de Economía y Finanzas y de los bancos centrales, frente al sistema actual, en el que esto no es posible al tener carácter de residente. Por otro lado, la eliminación del componente político en la toma de decisiones, ya que al delegar las decisiones operativas en un comité de gestión independiente se evitaría que primasen los criterios políticos, como ocurrió a juicio de los autores durante el rescate a Grecia, en el que prevaleció la visión de los países europeos dado su mayor peso.

Aunque este comité de gestión sería independiente, tendría que informar al directorio no residente de las decisiones adoptadas en reuniones periódicas, donde se supervisaría el cumplimiento del mandato, que vendría definido en los estatutos del FMI, dejando total autonomía a los miembros del comité para que decidan la estrategia para alcanzarlos. Adicionalmente, el comité de gestión estaría sometido a un segundo control y su gestión sería supervisada por la oficina independiente de evaluación, que tendría que ser reforzada para asegurar su independencia. 


\subsubsection{Nuevo instrumento de préstamo}

El nuevo instrumento iría dirigido a los países con fundamentos sólidos que se vean afectados por una crisis. Este instrumento lo proponen por la escasa demanda que en la actualidad tienen los instrumentos de préstamo precautorio, y que se debe, principalmente, al miedo que tienen los países de solicitar la precalificación, ya que los mercados pueden interpretarlo de forma negativa y se podría desencadenar la propia crisis que se quiere evitar.

Los recursos para financiar este instrumento se obtendrían por un procedimiento muy sencillo. Se emitirían derechos especiales de giro (DEG), que serían asignados a todos los países en función de sus cuotas; esta decisión únicamente requeriría una mayoría cualifica$\mathrm{da}^{3}$. Posteriormente, se haría una llamada para que los países realizasen contribuciones voluntarias de los nuevos DEG asignados, que serían utilizadas para financiar el nuevo instrumento de préstamo.

El rápido acceso, y sin condiciones, sería la principal ventaja que ofrecería este instrumento. Precisamente para garantizar la rápida aprobación de este préstamo es fundamental la labor de supervisión que el FMI realiza en el marco del proceso de las consultas del artículo IV ${ }^{4}$ y gracias a la cual el Fondo dispone de la información necesaria para valorar si un país

\footnotetext{
3 Las decisiones importantes tienen que ser adoptadas por una mayoría cualificada de, al menos, el 85 por 100 del total de votos. Esto supone que EEUU, en la práctica, y los países de la UE (si votasen de forma cohesionada) tienen derecho de veto porque poseen una cuota superior al 15 por 100.

4 Una de las funciones centrales del FMl es la supervisión del sistema monetario internacional a escala mundial, regional y nacional. El proceso de las consultas del artículo IV es como se conoce a la supervisión bilateral, que consiste en visitas anuales a los países miembros en las que un equipo del FMI se entrevista con representantes del Gobierno y del banco central, funcionarios y parlamentarios, así como con representantes sindicales, del sector financiero y empresarial y de la sociedad civil. Los principales temas tratados en dicho intercambio se refieren a las políticas cambiaria, monetaria, fiscal y macrofinanciera, así como a reformas macroeconómicas estructurales.
}

cumple con los requisitos para obtener acceso a este préstamo. Tras ser aprobado dicho acceso, se enviaría una misión al país para evaluar si las condiciones han empeorado de forma considerable respecto a la última consulta del artículo IV y, en caso de que lo hayan hecho, el préstamo se transformaría en un Acuerdo de Derecho de Giro 5 .

Se trata de un préstamo que no puede ser renovado, tendría una duración máxima de tres a seis meses, y la cantidad requerida podría tener que limitarse, ya que al ser un préstamo que se caracteriza por su rápido acceso, los recursos disponibles podrían no ser suficientes.

El funcionamiento de este instrumento sería similar a los swaps bilaterales, pero con la ventaja de que no lo estaría otorgando un solo banco central, sino un grupo de países, por lo que el riesgo sería menor, aunque con el inconveniente de que los propios bancos centrales implicados no adoptan la decisión final de otorgarlo o no, sino que sería el comité de gestión el que tomaría esta decisión.

A veces el propio anuncio de la concesión del instrumento a un determinado país puede bastar para tranquilizar a los mercados, sin que el país tenga que acceder efectivamente a los recursos, ya que pondría de manifiesto que el FMI respalda las políticas y la solidez de la economía del país. Pero en caso de que tuviese que acceder a los recursos, el país demandante del instrumento simplemente tendría que solicitar la conversión de los DEG por monedas convertibles.

Con esta alternativa ya no sería necesario que los países con fundamentos sólidos mantengan una cuantía elevada de reservas, $\triangleright$

\footnotetext{
5 El Acuerdo de Derecho de Giro (Acuerdo Stand-By) es el instrumento típico de acceso efectivo (no precautorio) empleado por el FMI para otorgar préstamos a los países de mercados emergentes y avanzados. Los países de bajo ingreso utilizan los instrumentos concesionales.
} 
con el coste y las ineficiencias que esto conlleva, para hacer frente a tensiones puntuales de balanza de pagos.

\subsection{El informe del grupo de personas de reconocido prestigio del G20}

En el marco del G20, los ministros de Economía y Finanzas en abril de 2017 acordaron la creación de un grupo de expertos formado por personas de reconocido prestigio (eminent persons) y con el mandato concreto de elaborar un informe con propuestas específicas para adaptar la arquitectura financiera internacional al nuevo contexto económico y la revisión del papel que en el mismo debería jugar el G20.

Este informe fue presentado a los ministros de Finanzas y Economía en las reuniones anuales que tuvieron lugar en octubre de 2018 en Bali, y la aplicación efectiva de sus recomendaciones será discutida a lo largo de 2019 en el marco del G20, aunque las propuestas específicas en que se concreten estas discusiones tendrán que ser finalmente negociadas y aprobadas por los órganos de gobierno de las diferentes instituciones implicadas.

De todas las propuestas recogidas en este informe, en este artículo abordaremos únicamente las relacionadas con el FMI, en concreto sobre su sistema de gobernanza y a sus funciones de asesoramiento, supervisión y préstamo, dejando al margen las relativas a los bancos multilaterales de desarrollo y al G20.

Con el objetivo de que los países puedan beneficiarse de los flujos de capitales tanto domésticos como extranjeros, pero minimizando la volatilidad y el riesgo de que los inversores salgan del país a pesar de presentar unos fundamentos económicos sólidos, el informe propone una serie de medidas:
- Reforzar la coordinación de los diferentes programas de asistencia técnica dirigidos a fortalecer los mercados financieros domésticos, tanto desde un punto de vista regulatorio como institucional, y que son desarrollados por el FMI, el Banco Mundial y los bancos regionales de desarrollo.

- Ampliar el enfoque del FMI ${ }^{6}$ respecto a la gestión de capitales para que no solo se incluyan medidas dirigidas a liberalizar la gestión de capitales. En concreto, se propone que se realice un análisis en profundidad de los flujos de capitales desde el punto de vista tanto del país receptor como del país emisor. Por lo que, por un lado, se analizarían los factores internos que han influido en la entrada de capitales en un país y los que desencadenarían su salida, y, por otro lado, los factores que influirían en el retorno de los flujos de capitales al país de origen.

- Elaborar un menú de políticas económicas dirigido a los países emisores de capitales para que sus políticas internas no generen un impacto adverso en la economía internacional ${ }^{7}$. El seguimiento del impacto de las políticas internas de los países emisores se haría en el marco de la función de supervisión del artículo IV.

- La creación de un mecanismo de alerta temprana, el denominado mapa de riesgos $^{8}$, que identificaría y actualizaría los $\triangleright$

6 El FMI defiende la apertura a la inversión extranjera directa, ya que beneficia a casi todos los países, aunque los fundamentos económicos no sean sólidos. Pero, antes de liberalizar otros tipos de flujos, los países deben analizar si reúnen las condiciones mínimas que permiten aprovechar las ventajas netas de la globalización financiera sin estar expuestos a su volatilidad, y deben valorar si los controles de capitales tienen costes en términos de eficiencia.

Complementaría el trabajo que el FMI realiza en esta materia, que desde 2015 elabora notas temáticas.

8 El FMI ya realiza con carácter semestral ejercicios de alerta anticipada que evalúan los riesgos de baja probabilidad pero alto impacto para la economía mundial e identifican políticas para mitigarlos, supervisando a nivel nacional, regional y mundial el seguimiento de las conclusiones de estos ejercicios y de la aplicación de sus recomendaciones. 
riesgos susceptibles de afectar la estabilidad del sistema, que también incluiría un menú de políticas económicas y reformas para hacerlos frente. Además, se haría un seguimiento sobre el grado de preparación de los países en caso de que se materializasen los riesgos, y que se realizarían en el marco de las consultas del artículo IV.

También se proponen medidas similares al Informe de Ginebra, como el establecimiento de protocolos de coordinación entre el FMI y los acuerdos financieros regionales, dotar al FMI con suficientes recursos, la creación de un nuevo instrumento que permita a los países con fundamentos sólidos acceder a préstamos de forma ágil en momentos de crisis y la reforma del sistema de gobernanza, delegando un mayor poder en el equipo encargado de la gestión para que el Directorio Ejecutivo del FMI no tenga que decidir sobre cuestiones operativas.

Como se ha apuntado, en el actual contexto económico complejo y a la vista de las reformas que reclaman los expertos la Decimoquinta Revisión General de Cuotas del FMI adquiere especial relevancia.

\section{La reforma de las cuotas del FMI}

El FMI es un fondo común ( $p o o l)$ de divisas de los países miembros, que se aportan en forma de cuotas (una parte en moneda propia y otra en derechos especiales de giro, DEG, o en monedas consideradas de libre uso — dólar estadounidense, euro, renminbi, yen o libra esterlina-). En el momento de incorporación de un país al Fondo, se le asigna una cuota que refleja, con carácter general, su posición en la economía global.

La cuota es importante puesto que determina la relación financiera (y de gobierno) del país con el Fondo. Por un lado, determina el importe máximo de recursos de aportación obligatoria al FMI. Por otro, determina en buena medida el poder de voto en la institución (se establece un número de votos básicos por país, a los que se suman votos por volumen de cuota aportada). Por último, el acceso máximo de un país a la asistencia financiera del Fondo se establece en términos de su cuota (por ejemplo, los acuerdos precautorios pueden alcanzar un máximo de un 145 por 100 de la cuota), aunque en circunstancias excepcionales el acceso puede ser superior.

El volumen total y la asignación entre países de estas cuotas se revisan cada cinco años. Actualmente se está negociando la revisión número quince de la historia del Fondo. Se trata de uno de los principales debates que tendrá lugar el próximo año, con el objetivo de llegar a un acuerdo durante las próximas reuniones de primavera de 2019 y no más tarde de las reuniones anuales del próximo octubre, en cumplimiento del mandato de la Junta de Gobernadores, de diciembre de 2016.

Las directrices que se tendrán en cuenta en las negociaciones son las que aparecen en el último comunicado del Comité Monetario y Financiero Internacional, en el que los países reafirman su «compromiso de que el FMI siga siendo una institución sólida, con una dotación adecuada de recursos y basada en cuotas, con el fin de preservar su función central en la red de seguridad del sistema financiero internacional» 9 . Además, se acuerda que la nueva fórmula que se utilice para el cálculo de las cuotas suponga un aumento de las cuotas de las economías dinámicas (en alusión indirecta a las $\triangleright$

9 Comunicado de la Trigésima Octava Reunión del Comité Monetario y Financiero Internacional: https://www.imf.org/es/News/Articles/2018/10/ 10/communique-of-the-thirty-eighth-meeting-of-the-international-monetaryand-financial-committee 
principales economías emergentes, los denominados BRIC —Brasil, Rusia, India y China- y en particular China), en función de su posición relativa en la economía mundial, aunque protegiendo a su vez la voz y la representación de los países miembros más pobres.

La experiencia de las últimas revisiones generales refleja que se trata de un proceso complejo, largo y políticamente difícil (requiere una mayoría cualificada del 85 por 100 de los votos, de modo que EEUU es el único país con poder de veto al ser su cuota superior al 15 por 100). Así, por ejemplo, en 2010 se acordó doblar las cuotas (14. evisión), si bien el aumento no fue efectivo hasta enero de 2016, cuando finalmente se consiguió que dicho acuerdo fuera ratificado por el Congreso de EEUU. Esto explica que en momentos de elevados riesgos globales, como la crisis financiera global estallada en 2008, se hayan puesto en marcha mecanismos adicionales para suplementar las cuotas del Fondo a través de los llamados «recursos prestados» (new arrangements to borrow, NAB, y préstamos bilaterales).

\subsection{Situación actual de los recursos y determinación de las cuotas}

Teóricamente, el Fondo cuenta con 960.000 millones de DEG en recursos, entre cuotas, $\mathrm{NAB}$ y bilaterales, que en la práctica se traducen en una capacidad de préstamo de 693.000 millones de DEG (en torno a un billón de dólares), teniendo en cuenta los fondos que se mantienen como reserva y los requisitos para activar los diferentes niveles de recursos.

- Cuotas: tras la entrada en vigor de la 14. revisión, las cuotas se doblaron y ascienden hoy a 477.000 millones de DEG.
- NAB: se trata de un instrumento de préstamo multilateral y voluntario para los países miembros del FMI, que actúa como segunda línea de defensa ante la aparición de necesidades de financiación. Al duplicar las cuotas con la entrada en vigor de la 14. ${ }^{\text {a }}$ revisión, se decidió reducir estas aportaciones (roll-back) a 182.000 millones de DEG, y se han renovado hasta 2022.

- Préstamos bilaterales: de carácter voluntario como los NAB, pero, a diferencia de estos, de naturaleza bilateral, constituyen la tercera línea de defensa ( $y$, por tanto, solo se activan cuando el acceso a las otras dos fuentes de recursos, cuotas y NAB, ya se ha visto agotado). Fueron introducidos en 2012 en un contexto de crisis y temor ante un posible aumento repentino de la demanda de asistencia financiera al FMI, que podría requerir recursos adicionales de manera urgente. Los actuales préstamos bilaterales (firmados en 2016) alcanzan una cuantía de 319.000 millones de DEG y estarán en vigor hasta 2019, con opción de prórroga por un año adicional hasta 2020.

Mientras que los recursos prestados (NAB y préstamos bilaterales) son voluntarios, la contribución de cada Estado miembro al FMI en forma de cuotas es obligatoria y su cuantía concreta está determinada por una fórmula acordada en 2008. Esta recoge una media ponderada del PIB (50 por 100 de la fórmula, del cual el 60 por 100 está fijado a precios de mercado y el 40 por 100, en paridad de poder adquisitivo), la apertura (30 por 100), la variabilidad (15 por 100) y las reservas (5 por 100). Se incorpora, además, un factor de «compresión» para reducir la dispersión entre países y $\triangleright$ 
minorar parcialmente el sesgo de la fórmula asociado al tamaño, generando una cierta redistribución de cuotas de los países más grandes hacia los demás.

\subsection{La Decimoquinta Revisión General de Cuotas: antecedentes y cuestiones a resolver}

Actualmente se trabaja en la 15. ${ }^{\text {a }}$ Revisión de Cuotas, que será una oportunidad para evaluar el tamaño y composición de los recursos del Fondo, así como avanzar en el proceso de reforma de su gobernanza (representatividad).

Las revisiones generales permiten medir la adecuación de los recursos del Fondo ante las posibles necesidades financieras de las economías, así como ajustar las cuotas para que estas recojan los cambios en la posición relativa de los países en la economía mundial. De hecho, existe una fuerte presión en los últimos años para incrementar el peso de los países emergentes (en detrimento de los avanzados) para corregir la falta de representatividad de la institución.

Las últimas reformas ya incluyeron medidas para tratar de atender estas reivindicaciones. Por ejemplo, en 2008, se realizó un aumento ad hoc de cuotas de 54 países que se consideraban infrarrepresentados y se triplicó el número de votos básicos, asignados por igual a todos los países miembros y que (como sucede con el factor de compresión en la fórmula) contribuyen a reducir la dispersión en el peso del voto según el tamaño de la cuota, favoreciendo a los países de menor tamaño. En 2010, con la 14. ${ }^{\text {a }}$ revisión general se doblaron las cuotas y se trasladó más del 6 por 100 del peso hacia países emergentes y economías en desarrollo. Así, China pasó a situarse como tercer país miembro del FMI, de cuyos diez primeros accionistas cuatro son países emergentes (por orden de tamaño de cuota: China, India, Rusia y Brasil). Esto se hizo compatible con el mantenimiento de las cuotas y el poder de voto de los países más pobres y de los más pequeños.

La actual revisión de cuotas, sin duda, deberá profundizar en este camino. Para ello, es necesario abordar cuatro aspectos:

- El tamaño óptimo del FMI. Esta es la primera cuestión a resolver. Ya en los años noventa comenzó a ponerse en duda que los recursos del FMl fuesen suficientes para hacer frente a las crisis; en concreto, las necesidades de México en 1994-1995 fueron muy superiores a la cantidad que podía tomar prestada del FMI, el 300 por 100 de su cuota. De hecho, se le acabó concediendo un préstamo de un 689 por 100 de su cuota.

Es cierto que los recursos del FMI se han visto más que triplicados desde el estallido de la crisis en 2008, si bien, entre 2019 y 2022, vence el 51 por 100 de estos recursos (los NAB y los préstamos bilaterales).

Tal y como refleja el Gráfico 1, el vencimiento de estos recursos, sin modificar las cuotas, supondría una merma significativa a la capacidad de financiación del Fondo ${ }^{10}$.

Esta situación exige tomar una decisión acerca del nivel deseable de recursos de la institución más allá de los citados vencimientos.

Hay países que son escépticos en cuanto a la necesidad de que se mantenga $\triangleright$

10 La FCC (Forward Commitment Capacity) es la medida de referencia del FMI de liquidez prestable, efectivamente, en los siguientes doce meses. Esta medida tiene en cuenta los recursos utilizables y los ingresos por recompra de divisas, menos las devoluciones previstas y un margen prudencial. 


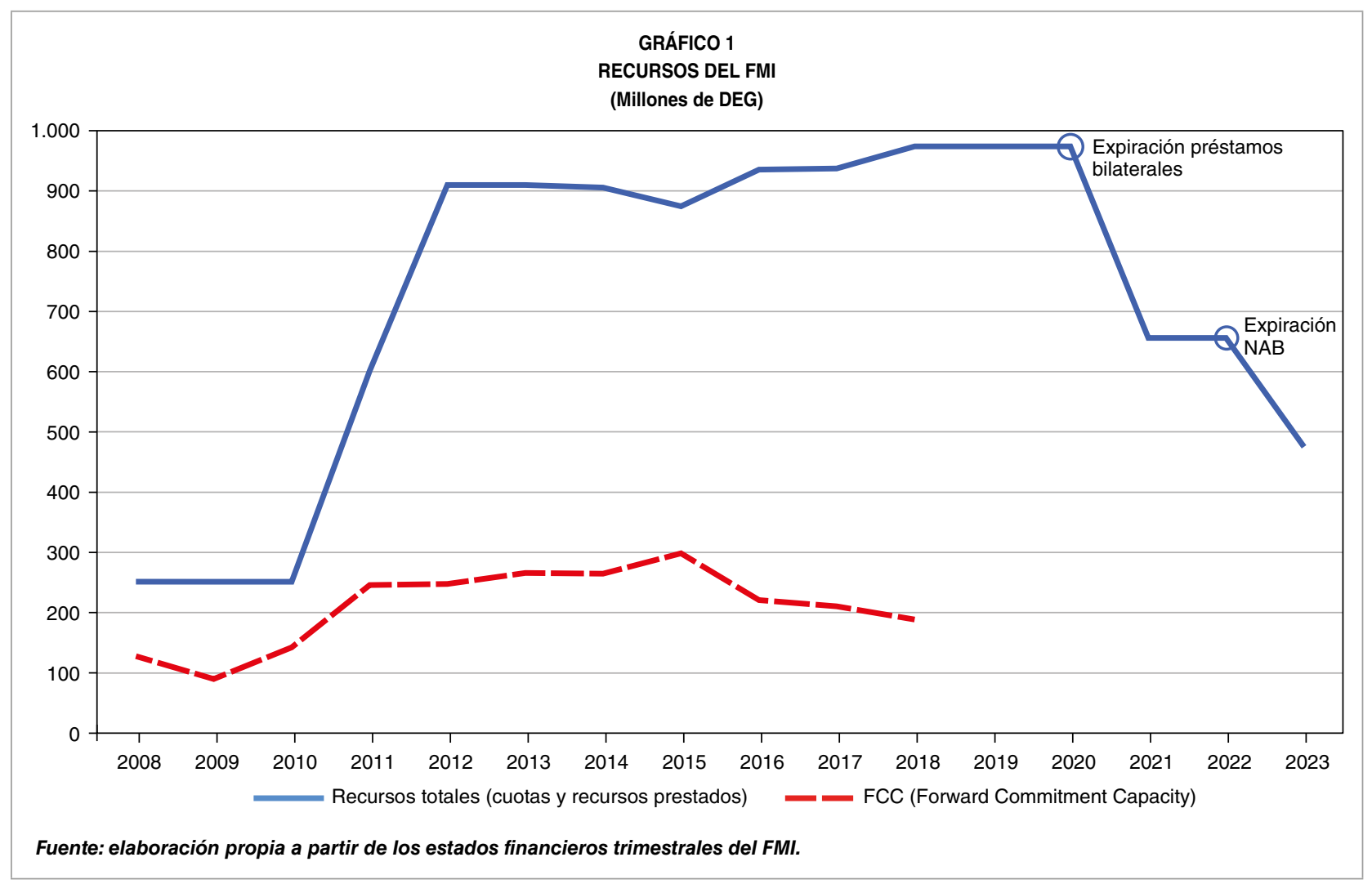

el tamaño actual del Fondo, teniendo en cuenta que este responde a un contexto de crisis ya superado y a la aparición en escena de una serie de acuerdos de financiación regionales (incluido el Mecanismo Europeo de Estabilidad) capaces de realizar parte de la labor de estabilización a nivel regional. Además, es cierto que, hasta la fecha, el volumen de fondos que suponen las cuotas ha sido suficiente para financiar los programas de préstamo.

Frente a ellos, muchos países, incluido el grueso de los emergentes, apoyan la necesidad de mantener los recursos actuales del Fondo. El argumento principal es que la mera existencia de un FMI fuerte aporta una señal potente que contribuye a la estabilidad del sistema financiero global. Además, una vez estalló la reciente crisis global, el Fondo necesitó de forma sobrevenida recursos adicionales y fue necesario articular mecanismos suplementarios de manera urgente. Las necesidades de financiación del reciente programa de Argentina han alcanzado un récord histórico (57.000 millones de dólares), y actualmente otros países emergentes (señaladamente, Turquía y Sudáfrica) y países de renta baja altamente endeudados están sufriendo tensiones en los mercados financieros y por consiguiente presiones sobre su balanza de pagos, con lo que se volvería a poner de manifiesto la importancia de contar con un FMl fuerte.

Aunque es difícil identificar un nivel óptimo de recursos del FMI, teniendo en cuenta los factores de riesgo (como los mencionados) y los que contribuyen a su mitigación (en particular, la reforma y refuerzo de los sistemas financieros $D$ 
tras la crisis global), son de interés los datos que arrojan los ejercicios de estrés.

Así, por ejemplo, el Banco Central Europeo (BCE, 2018) estima que una crisis internacional de carácter moderado daría lugar a una necesidad de financiación global de 2.258 millones de dólares, que se elevaría a 2.887 millones de dólares en caso de crisis severa. Teniendo en cuenta las reservas que mantienen los países y los recursos que podrían aportar los diferentes marcos de integración regional (como capas adicionales de seguridad de la red financiera internacional), se identifica que quedarían por cubrir unas necesidades financieras de 669 millones de dólares (986 millones de dólares en el caso más adverso).

A la vista de estas consideraciones, el mencionado vencimiento de los recursos prestados reviste especial importancia por su impacto sobre la capacidad financiera del FMI y hace necesario abordar la cuestión del tamaño de la institución.

- La composición de los recursos: cuotas vs recursos prestados. Con carácter general, hay acuerdo en torno a la deseabilidad de preservar la naturaleza de institución basada en cuotas del FMI, de manera que las contribuciones de los países a los recursos del Fondo estén directamente relacionadas con su peso en la economía mundial. Así se recoge en los últimos comunicados del Comité Monetario y Financiero Internacional.

No obstante, en la medida en que se decidiera no dejar menguar los recursos del FMI, hay que tener presente cuestiones de viabilidad política de un aumento significativo en las cuotas que pudiera afectar en gran medida a la presencia de los países avanzados (tradicionalmente, los más representados en la institución).

El ejemplo de la situación estadounidense es quizá el más claro para ilustrarlo: en la actualidad cuentan con capacidad efectiva de veto gracias a una cuota del 17,4 por 100. Un aumento de cuotas que redujera el peso de EEUU por debajo del umbral del 15 por 100, que les otorga esa capacidad, les obligaría a elevar la cuestión al Congreso. Para que mantengan este nivel, sin embargo, parece que el aumento máximo de cuota se situaría sobre el 7 por 100, en ningún caso suficiente para suplir el vencimiento de los recursos prestados.

Por este motivo, cobra relevancia el estudio en profundidad del diseño actual de los recursos prestados, originalmente pensados como instrumento temporal. Parece sensato que se estudien las posibilidades, puesto que, de decidirse efectivamente mantener los recursos del FMI, habrá que valorar las posibilidades que ofrecen estas herramientas y las vías de mejora a lo largo de 2019.

- La fórmula que define las cuotas. Existe cierto consenso a la hora de reconocer los méritos de esta fórmula, que estaría reflejando bien las realidades económicas y permitiendo mejoras de representatividad en el FMI, con lo que cabe esperar que la 15. a revisión no incorpore grandes novedades. No obstante, sí podría haber matices. Así, por ejemplo, los países avanzados (en particular, la UE) defienden que se maximice el peso del PIB a precios de mercado y la apertura, mientras que los emergentes prefieren el PIB en paridad del poder adquisitivo. De hecho, esta última variable se $\square$ 
incorporó a la fórmula en 2008 para dar respuesta a su petición, y en principio con carácter temporal. Además, se han identificado ciertos sesgos e imprecisiones de medición en la variable «apertura». No se cuestiona su importancia, en particular por consistencia con la principal misión de sus programas de asistencia financiera de hacer frente a problemas de balanza de pagos, si bien sí hay propuestas para tratar de corregir los sesgos hacia pequeñas economías muy abiertas y con grandes centros financieros (p.ej., Holanda, Bélgica, Luxemburgo, Suiza y Singapur).

Otra de las cuestiones que se plantea es si incorporar a la fórmula, de alguna manera, las aportaciones que se hacen por la vía de recursos prestados, de manera que estos sean determinantes también para la representación en el FMI.

En cualquier caso, conviene tener presente las diferencias entre la cuota teórica de los países (calculada conforme a la fórmula) y la que se observa en la práctica (que recoge inercias pasadas). Así, por ejemplo, EEUU tiene una cuota teórica del 14,7 por 100, si bien en la práctica concentra el 17,4 por 100 ya mencionado, de tal manera que es un país sobrerrepresentado. Es el caso también de otros países avanzados, incluidos Japón (6,5 por 100 actual frente al 5,1 por 100 teórico), Alemania (5,6 por 100 frente al 4,9 por 100$)$, Francia (4,2 por 100 frente al 3,1 por 100), Reino Unido (4,2 por 100 frente al 3,6 por 100), Italia (3,2 por 100 frente al 2,3 por 100) y España (de forma novedosa, con una cuota teórica del 1,7 por 100 y una representación en la práctica del 2 por $\left.100^{11}\right)$. Frente a estos, hay otros países infrarrepresentados respecto de lo que teóricamente les correspondería. EI caso más destacado es el de China, con una cuota teórica del 12,9 por 100 y un peso en la práctica del 6,4 por 100 .

- Reasignación de cuotas. Como se ha dicho, uno de los objetivos destacados de las revisiones generales de cuotas es introducir ajustes que permitan una mayor representatividad del FMI en función del peso de los países en la economía mundial y, con ello, una mejora de su legitimidad. Parte de esta tarea es la corrección de las diferencias entre las cuotas teóricas y calculadas de los diferentes países. Por ello, en caso de que se decida mantener el tamaño actual del Fondo, compensando el vencimiento de los NAB y los préstamos bilaterales, y que el correspondiente aumento de recursos se haga vía cuotas, habrá que decidir cómo se distribuyen estas.

Hay tres opciones para realizar esa distribución: (i) equiproporcionalmente, de tal manera que el reparto se haga conforme a las cuotas actuales y, por tanto, manteniendo el peso de las representaciones actuales (una opción poco factible, que ha perdido peso en las últimas revisiones y que es contraria al objetivo declarado de mejorar la representatividad); (ii) selectivamente, usando la fórmula (con los cambios que se puedan acordar), lo que supone reducir la $\triangleright$

11 Se trata de una novedad de la última revisión de cuotas en vigor desde 2016, puesto que, hasta entonces, España había sido tradicionalmente un país infrarrepresentado en el FMI (en parte debido a su incorporación tardía a la institución y en un momento, en concreto 1958, anterior al fuerte desarrollo económico del país, que ha tardado en tener un reflejo adecuado en nuestra cuota en el Fondo). Además, en los últimos años también se ha dejado sentir en la estimación de nuestra cuota teórica el impacto de la crisis sobre el PIB español. 
cuota de los países sobrerrepresentados a favor de los infrarrepresentados; (iii) o bien de manera ad hoc, incrementando la cuota de unos pocos países especialmente infrarrepresentados. A lo largo del tiempo, las revisiones han otorgado cada vez mayor importancia a los componentes selectivo y ad hoc de la distribución de las cuotas.

Por tanto, la revisión general de cuotas comporta diferentes aspectos y requiere decisiones complejas. Hasta la fecha se ha realizado mucho trabajo a nivel técnico, si bien las discusiones se encuentran todavía en una fase muy preliminar. Una de las cuestiones que se ha puesto sobre la mesa y que defiende, en particular, la UE, es que la revisión debe abordarse como «paquete integral», de manera que no habrá acuerdo sobre una parte de la revisión hasta que no lo haya sobre el conjunto. Parece sensato que no se tomen decisiones parciales sin que se pueda determinar cuál será el escenario final, si bien es cierto que hace más difícil que los avances sean visibles.

\section{Conclusiones}

En la actual coyuntura económica de mayores incertidumbres y empeoramiento de las perspectivas económicas, se hace especialmente urgente abordar la revisión del FMI, pero no solo para determinar si dispone de un nivel adecuado de recursos y mantener su naturaleza de institución basada en cuotas. Así, también es imprescindible realizar un análisis en profundidad sobre la adecuación de las diferentes herramientas con las que cuenta el FMI para ejercer sus funciones de supervisión, préstamo y fortalecimiento de capacidades.
Este proceso de revisión es fundamental para asegurar que el FMI continúe desempeñando un papel protagonista en la red de seguridad del sistema financiero internacional, y 2019 es el año decisivo para su conclusión. Aunque es cierto que hasta la fecha los avances han sido muy limitados, y el éxito de la revisión depende en último término de la posición aún por dilucidar de EEUU, el éxito en cerrar un acuerdo para la revisión de los recursos del Grupo del Banco Mundial en las asambleas de primavera de 2018, con el apoyo estadounidense en la recta final de las negociaciones, permite que seamos moderadamente optimistas respecto a la posibilidad de que se alcance un acuerdo también en el caso del FMI dentro del plazo límite de las asambleas anuales de octubre de 2018.

\section{Bibliografía}

[1] BCE (2018). «A quantitative analysis of the size of IMF resources». Occasional Paper Series, n. .213 , octubre.

[2] COMITÉ MONETARIO Y FINANCIERO INTERNACIONAL (2018): Comunicado de la Trigésima Octava Reunión del Comité Monetario y Financiero Internacional. 13 de octubre 2018. Bali (Indonesia). Disponible en: https://www.imf. org/es/News/Articles/2018/10/10 communiqueof-the-thirty-eighth-meeting-of-the-international-monetary-and-financial-committee

[3] DE GREGORIO, J.; EICHENGREEN, B.; ITO, T. y WYPLOSZ, C. (2018). «IMF Reform: The Unfinished Agenda». Geneva Reports on the World Economy, n.. 20.

[4] G20 EMINENT PERSONS GROUP (2018, octubre). Report of the G20 Eminent Persons Group on Global Financial Governance. Disponible en: https://www.globalfinancialgovernance.org/report-of-the-g20-epg-on-gfg/

[5] GALLEGO, S.; L'HOTELLERIE-FALLOIS, P. y LÓPEZ-VICENTE, F. (2018). «El Fondo Monetario Internacional y su papel como $D$ 
Subdirección General del Sistema Financiero Internacional

garante de la estabilidad financiera global». Boletín Económico ก.ำ 4, noviembre, Banco de España.

[6] LAGARDE, C. (2018): Un nuevo panorama económico, un nuevo multilateralismo. Discurso para las Reuniones Anuales de 2018. Bali (Indonesia), octubre. Disponible en: $h t t p s: / / w w w$. imf.org/es/News/Articles/2018/10/11/ sp101218-new-economic-landscape-newmultilateralism 